\title{
La procréation : une fonction naturellement inhibée
}

\author{
Jacques Testart
}

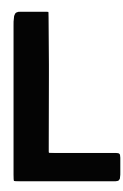

es limites naturelles à la procréation humaine ne sont qu'imparfaitement exprimées par les indices de fécondité: le constat démographique que chaque couple (deux individus) ne procrée actuellement que 1,7 enfant correspond à une évaluation psychosociologique plutôt que biologique. Médicalement, on constate que $15 \%$ environ des couples rencontrent de sérieuses difficultés pour réaliser leur projet procréatif [1], ou encore que le risque mensuel de conception n'est que de $20 \%$ à $30 \%$ pour un couple fertile [2]. Cette aptitude à procréer est bien réduite au regard de celle connue chez l'animal, mais notre faible fécondité ne résulte pas du progrès médical, supposé favoriser les géniteurs peu fertiles, car ce progrès n'a pu bénéficier qu'à la dernière génération. Cependant, ces données ne rendent pas compte d'un phénomène plus général qui concerne l'aptitude normale à procréer: aucune propriété biologique n'est naturellement soumise à autant d'empêchements variés que la fonction de procréation, empêchements qui concernent de nombreux êtres vivants (en particulier tous les mammifères), mais qui atteignent l'espèce humaine à un degré supérieur.

On pourrait, en exagérant à peine, considérer que des obstacles à effet contraceptif sont constitutifs de l'état naturel ; la preuve en est que la levée de ces obstacles par des procédés artificiels crée une situation inédite où $\mathrm{m} / \mathrm{s} n^{\circ} 3$, vol. 11 , mars 95 la procréation devient plus performante qu'elle ne le fut jamais pour aucun couple. L'assistance médicale à la procréation (AMP) permet, comme l'assistance à d'autres fonctions biologiques, de corriger des carences individuelles, mais seule l'AMP est capable d'intensifier une fonction naturelle jusqu'à un niveau d'efficacité jamais connu. Ainsi, le traitement de déficiences respiratoires ou digestives n'aboutit pas à augmenter, jusqu'à les dépasser, les caractéristiques normales de l'espèce, quant à la capacité respiratoire ou l'assimilation des aliments. Par ailleurs, il est vrai que si l'usage de la loupe améliore la performance visuelle, c'est par une action qui ne concerne pas l'œil, de même que l'avion ne fait pas de l'homme un oiseau.

Or, les interventions biomédicales transforment tous les aspects de la procréation en conférant à l'espèce des propriétés nouvelles, et aussi des performances inédites. L'interprétation triviale d'une éventuelle inhibition à procréer l'assimilerait à une inhibition psychologique de la sexualité. Sans nier cette composante, nous voulons envisager ici les empêchements physiologiques successifs qui affectent la capacité d'avoir des enfants.

\section{Age de fertilité : des années d'inhibition}

L'aptitude à la fertilité n'apparaît qu'à la puberté dans les deux sexes et cesse à la ménopause chez la femme, tandis qu'elle diminue progressivement avec la sénescence chez l'homme. Ces limites naturelles pourraient théoriquement être transgressées par l'établissement de la procréation, avant ou après la période fertile. Ainsi les gonades du fœus, ou de l'impubère, recèlent des cellules germinales susceptibles d'évolution immédiate, voire d'engendrer une descendance (figure 1): des follicules primordiaux, prélevés dans les ovaires de souris nouveau-nées, sont capables de reconstituer un ovaire fonctionnel chez une femelle adulte stérilisée, et les ovocytes qu'ils contiennent peuvent réaliser la maturation méiotique et être à l'origine de souriceaux normaux après fécondation de la femelle-hôte [3].

Chez le mâle, le noyau (diploïde) de la spermatogonie, prélevé dans le testicule fœtal et greffé dans un ovocyte énucléé, permet le développement embryonnaire au moins jusqu'au stade blastocyste [4]. Dans plusieurs espèces de mammifères domestiques, on a pu obtenir la descendance de femelles impubères en transplantant, dans l'utérus de femelles adultes, des embryons générés artificiellement, in vivo ou in vitro; on sait aussi hâter l'âge de la puberté par des manipulations hormonales. Dans l'espèce humaine, c'est seulement la prolongation de la période fertile qui a fait l'objet des efforts médicaux (cela confirme que les propositions biomédicales n'arrivent que par, ou au-devant, de la demande, le fœtus ou l'enfant ne pouvant être demandeur de 
procréation...). On sait ménager une descendance à l'homme au-delà de la période fertile, ou même de la mort, grâce à la conservation de sa semence par congélation. Chez la femme, pourvu qu'on dispose d'un embryon (figure 2) issu de l'ovule produit par une donneuse ou de son propre embryon congelé, l'utérus post-ménopausique s'est avéré capable d'assurer la grossesse et des enfants sont nés de patientes déjà grand-mères... L'aptitude des femelles à être fécondées et à mener la gestation est un facteur crucial pour la fécondité d'une espèce chez les mammifères. Aussi l'amplitude du stock des cellules germinales dans les ovaires reflète le degré du potentiel procréatif. Il est significatif que ce stock diminue avant même la naissance, au contraire de ce qui arrive chez le mâle, sexe très peu responsable de la fécondité, sauf quand la structure sociale ou la culture favorise les couples stables. Ce sort fait à l'ovogenèse plutôt qu'à la spermatogenèse a pour conséquence d'inhiber la procréation, phénomène qui semble institué depuis longtemps dans l'évolution.

Il apparaît donc que, si la période efficace de fertilité se trouve confinée à environ la moitié (chez la femme), ou les trois quarts (chez l'homme) de la vie biologique, c'est par des restrictions naturelles qui ne concernent pas la totalité du processus procréatif. Ces restrictions sont d'origines diverses et peuvent être court-circuitées à l'aide d'artifices qui permettent d'obtenir une descendance avant ou après l'âge "normal " de procréation. Elles peuvent aussi être contournées par la contribution à la procréation de personnes étrangères au couple (donneurs de gamètes ou d'embryons, mères-porteuses).

\section{Période féconde : des jours d'inhibition}

L'espèce humaine appartient à la catégorie minoritaire des mammifères chez lesquels l'activité sexuelle, et donc procréatrice, n'est pas saisonnière; elle peut donc concevoir des enfants tout au long de l'année. Cependant, c'est encore la femme qui dité, par l'existence de rythmes physiologiques ménageant de larges plages stériles au sein de cycles potentiellement fertiles. Les rapports sexuels sont rarement féconds, sauf si leur moment est calculé dans un but de procréation, car la production d'un embryon ne peut arriver que par la conjonction de ces rapports avec l'ovulation. Ainsi, compte tenu de la durée de survie des gamètes (moins de deux jours pour l'ovule et environ cinq pour le spermatozoïde), la période féconde féminine équivaut seulement au quart de la durée du cycle menstruel, et donc à environ $10 \%$ de la vie biologique.

Il existe, chez les femelles animales, un dispositif comportemental qui, en n'autorisant l'accouplement que dans la période utile de l'ovulation, augmente la probabilité du succès procréatif des rapports sexuels. Un tel dispositif, absent dans l'espèce humaine, est surtout efficace si la femelle réceptive est soumise aux assauts rapprochés de plusieurs mâles, situation inusuelle dans nos sociétés. C'est seulement chez les primates supérieurs, dont nous sommes, qu'il n'existe pas de repère comportemental de la période fertile chez la femelle. Ainsi, par la rareté des jours potentiellement féconds, et par leur caractère masqué, la physiologie humaine est remarquablement malthusienne. Grâce à des manipulations hormonales du cycle féminin, en particulier en inhibant l'action hypophysaire sur la sphère génitale avec des agonistes ou antagonistes du GnRH, le rythme cyclique peut être modifié: on sait induire l'ovulation au jour choisi plusieurs mois à l'avance. Cette "maîtrise " du fonctionnement ovarien doit aussi être capable de réduire certaines stérilités psychogènes puisque la gonade se trouve ainsi privée de l'influence cérébrale. En outre, des techniques diagnostiques (hormonales ou échographiques) permettent d'identifier le jour de l'ovulation spontanée aussi précisément que réalisé par l'exhibition de l'œstrus ( «chaleurs») dans les espèces animales... Or, quand les rapports sexuels ou l'insémination artificielle sont pratiqués au moment ainsi médicalement défini, les résultats indiquent que la fécondité est améliorée.

\section{Production des gamètes : gaspillage et restrictions}

Comme déjà évoqué, le stock de cellules germinales peuplant l'ovaire est maximum avant même la naissance et diminue progressivement jusqu'à la ménopause, cette diminution étant le résultat de l'atrésie bien plus que de l'ovulation. Environ cinq millions d'ovogonies peuplent les ovaires fœtaux dont 500000 persistent (sous forme d'ovocytes) à la naissance, parmi lesquels moins de 500 évolueront jusqu'à l'ovulation (figure 3). Rien n'autorise à penser que ces ovules sont sélectionnés selon des critères de normalité puisque de nombreux gamètes élus $(30 \%$ selon certaines évaluations) portent des anomalies chromosomiques [5], et que d'autres, en proportion inconnue, recèlent des défauts géniques. Cet énorme gâchis germinal, absent chez le mâle, induit une limitation puissante de la fécondité, non seulement parce que l'épuisement des réserves ovariennes signe la ménopause, mais aussi parce que le nombre d'ovules émis lors de chaque cycle se trouve restreint à l'unité. Le déterminisme final de cette ovulation unique, achèvement de la gabegie, commence à être connu ; ce serait un avantage infinitésimal (tel que la proximité de l'irrigation sanguine pourvoyeuse d'hormones) qui singularise le follicule ovulatoire (et donc l'ovocyte qu'il contient) parmi une dizaine de candidats comparables, deux semaines avant l'ovulation. Dès son élection, ce follicule exerce une action dominatrice sur les concurrents, survivants comme lui de dizaines d'années de déplétion au cours desquelles la plupart des structures équivalentes furent éliminées.

Pourtant la monoovulation n'est pas une fatalité et, depuis trente ans, on sait aussi remédier à cette restriction. Les traitements de stimulation ovarienne sont capables d'amener dix follicules en moyenne (et jusqu'à quatrevingts!) à l'ovulation (figure 4). Des recherches actuelles pourraient produire le même résultat sans administration exogène d'hormones gonadotropes (à effet FSH) mais, logiquement, en bloquant l'action inhibitrice de la follicle regulatory protein [6]. Ce se- 
rait là une démonstration exemplaire : à l'issue du gaspillage initial, l'ovulation unique résulte de phénomènes ultérieurs, limitant encore le potentiel ovarien.

Chez l'homme, des travaux récents ont révélé que de nombreuses protéines, sécrétées par le testicule ou l'épididyme, sont associées au spermatozoïde et interviennent dans les mécanismes de reconnaissance de l'ovule et d'attachement à ses enveloppes. La plupart de ces protéines favorisent la fécondation mais certaines semblent avoir une fonction inhibitrice sur l'attachement des gamètes [7], comme pour entraver la réalisation de la fécondation.

\section{Rencontre des gamètes : une réunion après handicaps}

Même quand le jour de l'insémination (naturelle ou artificielle) coïncide avec celui de l'ovulation, les chances de fécondation restent faibles dans l'espèce humaine. Car des handicaps topologiques et chronologiques empêchent la rencontre des gamètes dans les conditions optimales. Pour gagner le voisinage de l'ovule, les spermatozoïdes doivent réussir une course d'obstacles qui concourent indiscutablement à une fonction sélective. Par la suppression artificielle de ces obstacles, on favorise la rencontre des gamètes et la création d'un embryon. Il en est ainsi à l'occasion de l'insémination intra-utérine et, plus encore, de la fécondation in vitro (FIV) ou du transfert des gamètes dans la trompe (GIFT). Ces techniques d'AMP agissent de deux façons [8] : d'une part, elles suppriment tout ou partie des handicaps qui gênent la remontée des spermatozoïdes dans les voies génitales féminines : distance, structure physique (mucus cervical), nature chimique. Ainsi, un effectif élevé de gamètes mâles se trouve confronté à un ou quelques ovules. D'autre part, ces techniques exigent une précision chronologique qui évoque celle existant chez les mammifères dont l'ovulation est provoquée par le rapport sexuel (ex.: lapine, chatte) : c'est au moment où leur aptitude à la fécondation est maximum que les gamètes $\mathrm{m} / \mathrm{s} n^{\circ} 3$, vol. 11 , mars 95 se trouvent en contact, ce moment étant défini avec une précision de quelques heures seulement. La FIV peut réussir parfaitement cette gageure puisque l'ovule est recueilli dès que la maturation méiotique est réalisée (30 à 36 heures après injection de la gonadotropine hCG), et que les spermatozoïdes sont isolés du sperme fraîchement émis. Si la FIVETE (FIV et transfert d'embryon) a vite élargi ses indications au-delà des altérations tubaires, c'est que, par les propriétés énoncées cidessus, elle s'est avérée plus performante que d'autres traitements ou que les conditions naturelles, dans des cas variés d'infécondité, en particulier ceux que provoquent les déficiences du sperme. Toutefois, le score moyen de la FIVETE (15 accouchements pour 100 cycles d'essai) n'étant pas supérieur au score moyen de la procréation humaine, son succès thérapeutique ne démontre pas qu'aurait été ainsi levée une inhibition naturelle à la procréation, telle qu'elle existerait chez des couples normaux. C'est seulement par l'irruption de la fécondation assistée que cette démonstration vient d'être faite.

\section{Fécondation : I'inflation des mesures sécuritaires}

Comme s'il y avait un péril d'hybridation, chaque sexe de chaque espèce produit des gamètes qui ne peuvent s'associer qu'aux gamètes de l'autre sexe de la même espèce. Les choses paraissent relativement simples du côté féminin où la zone pellucide, enveloppe externe de l'ovule, porte une glycoprotéine nommée ZP3 [9], différente d'une espèce à l'autre, et reconnue seulement par le spermatozoïde homospécifique. Le contrôle identitaire pourrait s'arrêter là mais, pour des raisons encore obscures, de nombreuses autres molécules interviennent dans la liaison gamétique: ce sont des dizaines de protéines du spermatozoïde qui paraissent impliquées dans son attachement à la zone pellucide, puis à la membrane plasmique de l'ovule. On conviendra alors que le système est fragile, comme il arrive quand on augmente les dispositifs sécuritaires.
Ainsi, des spermatozoïdes, par ailleurs normaux à tous égards, peuvent s'avérer inféconds par incapacité à pénétrer l'enveloppe de l'ovule [10]. Une astuce récente consiste à injecter le spermatozoïde dans le cytoplasme ovocytaire (intracytoplasmic sperm injection, ICSI) et, en déjouant ces handicaps, elle produit des résultats étonnants [11] (figure 5). L'ICSI est aussi capable de féconder l'ovule avec des spermatozoïdes non éjaculés (épididymaires, testiculaires), et même avec des spermatides [12]. Non seulement l'ICSI permet la fécondation quand le sperme ne contient aucun spermatozoïde mobile, mais son succès dans ces cas extrêmes s'avère supérieur à celui de la FIV quand l'homme est fécond, ou de la procréation naturelle chez les couples normaux [13].

L'invention de la fécondation assistée apporte la preuve la plus éclatante que la procréation artificielle peut être plus efficace qu'elle ne l'est naturellement. Si de tels mécanismes répressifs ont été sélectionnés, ce pourrait être pour assurer la qualité du conceptus, mais la vérification que les enfants naissent normaux après ICSI [14] ne soutient pas cette hypothèse. Alors, il s'agirait d'un dispositif complémentaire à ceux déjà décrits, et dont la fonction serait encore de limiter la fécondité de l'espèce.

\section{Développement : synchronie, singleton et période réfractaire}

Après s'être étonné de l'aptitude de l'ICSI à produire des embryons, malgré l'incompétence du sperme, on est frappé de constater la meilleure viabilité des embryons ainsi produits, par rapport à ceux produits en FIV "classique" [ 14]. Il faut peut-être y voir une ultime sophistication chronologique : après le contrôle médical du jour puis de l'heure de l'insémination, on est arrivé à en contrôler l'instant précis puisque l'acte biologique d'ICSI annule tout délai pour l'intrusion du noyau spermatique au sein de l'ovoplasme. Les phases ultimes de la fécondation pourraient s'en trouver optimalisées. Cependant, d'autres facteurs encore incon- 


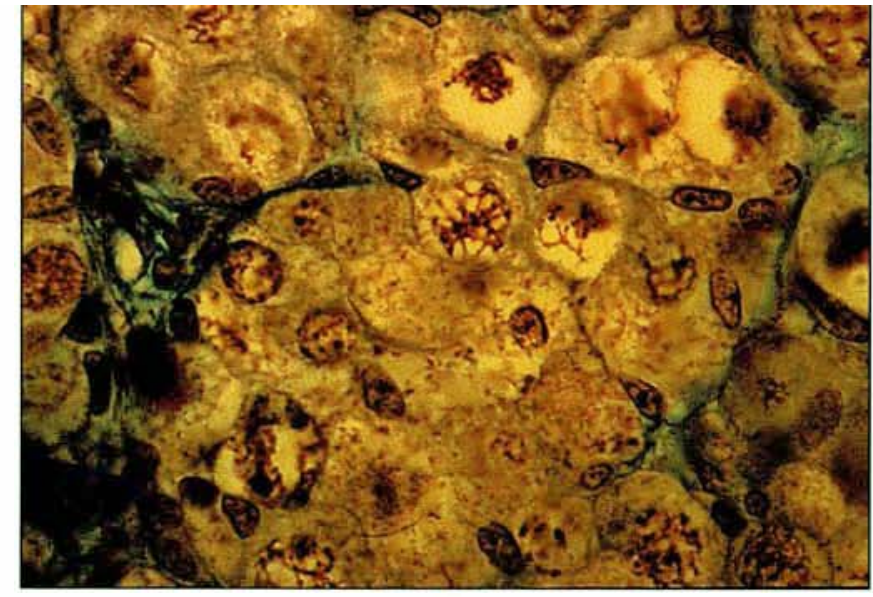

Figure 1. Coupe dans un ovaire de fœtus humain âgé de 5 mois. Les ovocytes commencent la prophase méiotique qui s'arrêtera au stade diplotène latteint avant la naissance) et ne reprendra que le jour précédant l'ovulation (mais la majorité de ces ovocytes aura disparu entre temps). (Photo: CNRI).

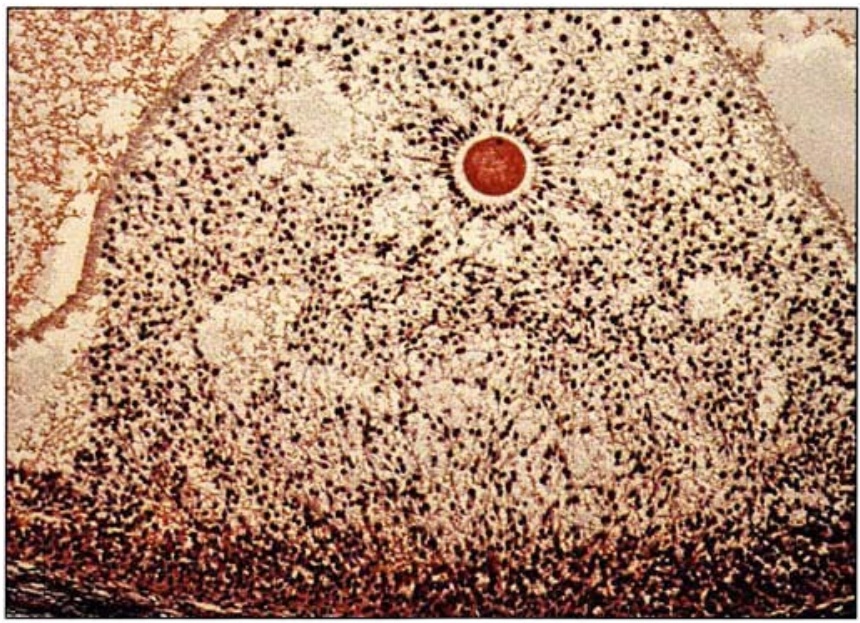

Figure 3. Ovocyte humain juste avant l'ovulation. Au sein du massif de cellules folliculaires (corona radiata), et à l'intérieur de la zone pellucide, on distingue l'ovocyte, dont le noyau a disparu (stade métaphase de la $2^{e}$ division méiotique) et, latéralement, le premier globule polaire. (Photo: CNRI).

Figure 4. A et B. Coupe dans un ovaire de singe après stimulation avec des hormones gonadotropes. De nombreux grands follicules sont visibles, contrairement à ce qui arrive dans un cycle non stimulé.

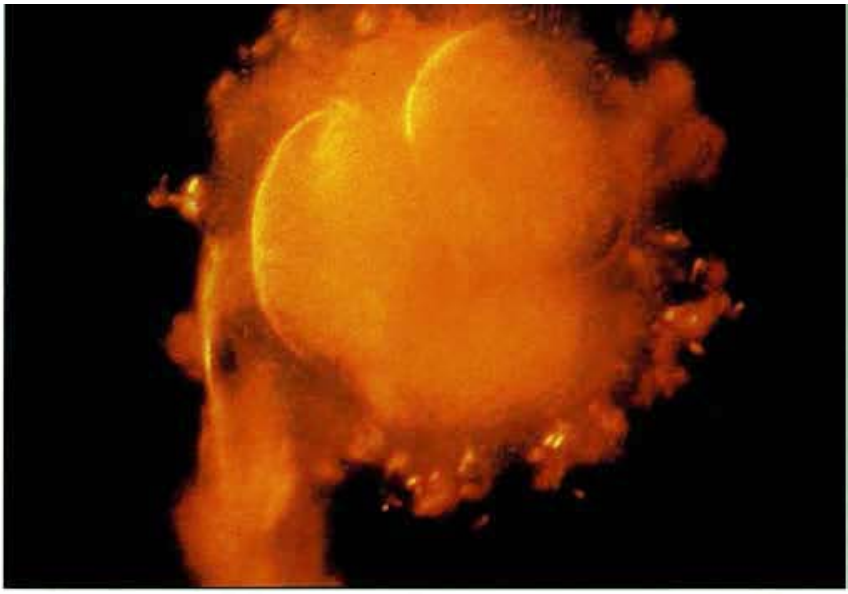

Figure 2. Embryon humain âgé de 2 jours après fécondation in vitro. La lumière rasante fait apparaitre trois des quatre blastomères. En périphérie persistent des cellules folliculaires. (Photo: CNRI).
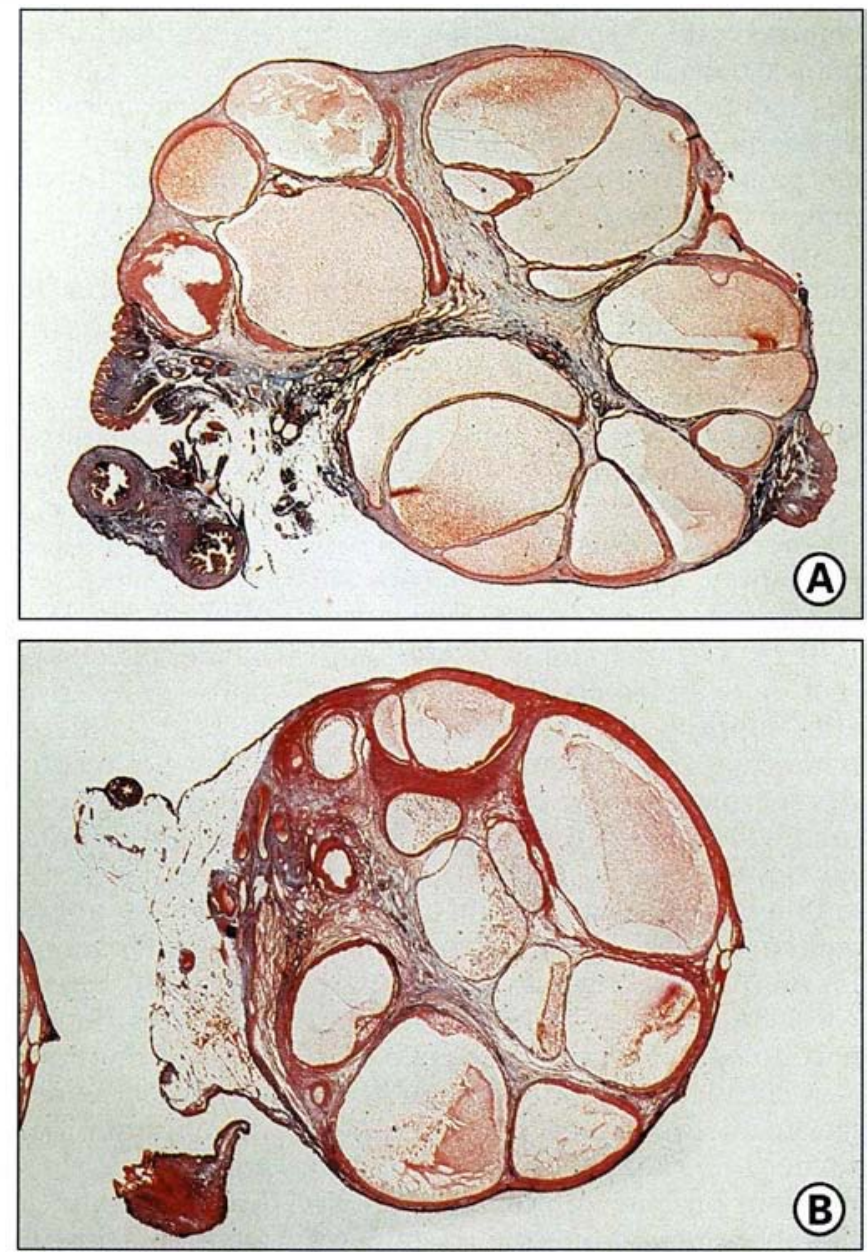

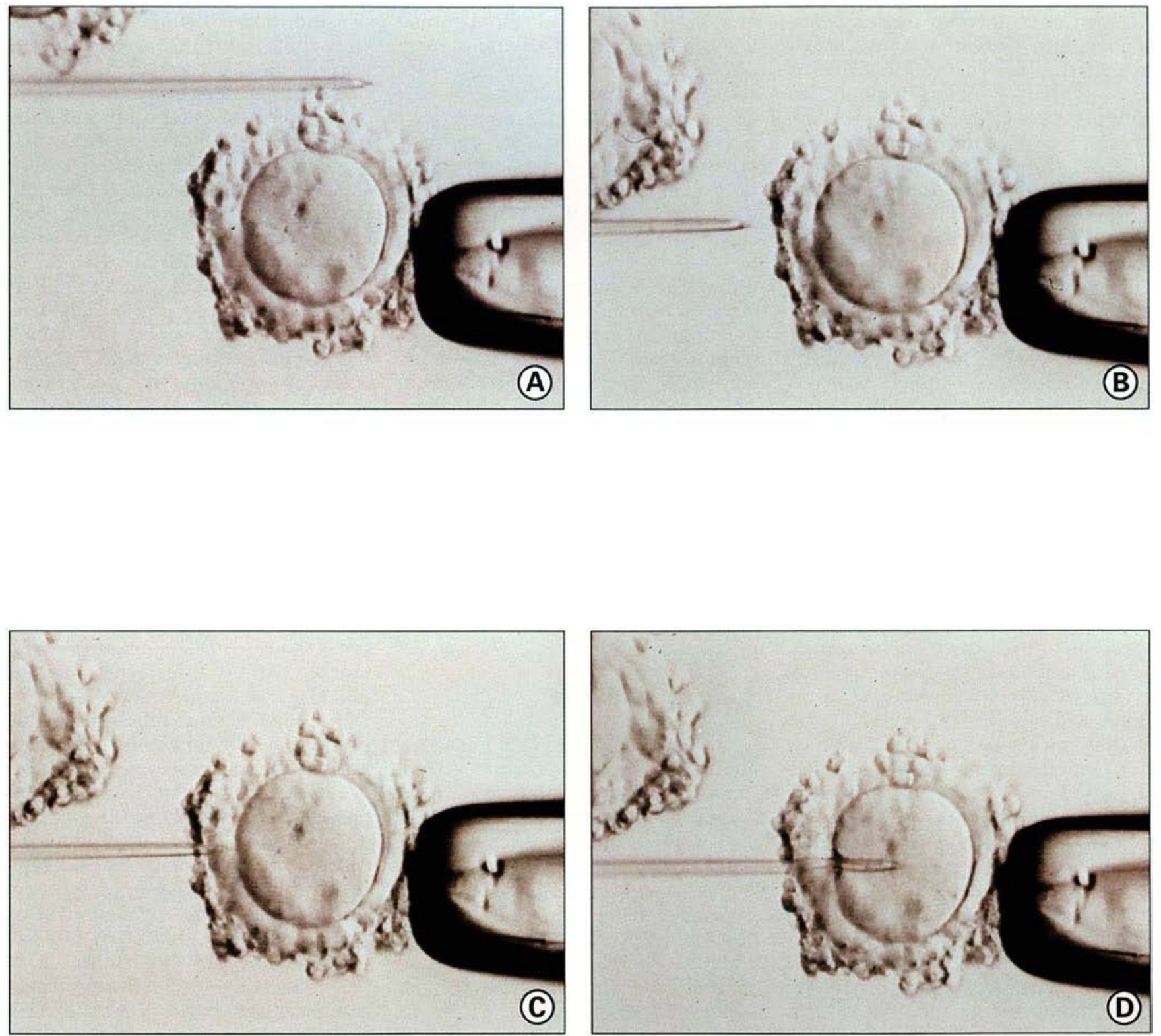

Figure 5. Technique de micro-injection du spermatozoïde dans le cytoplasme de l'ovule (ICSI: intracytoplasmic sperm injection). A. L'ovule est maintenu par aspiration à l'aide d'une grosse pipette. B. La petite pipette s'apprête à pénétrer les enveloppes ovulaires. C. Les cellules recouvrant l'ovule et l'enveloppe externe (zone pellucide) sont traversées. D. La pipette d'injection arrive au centre de l'ovule et va y déposer le spermatozoïde. (Photo: CNRI). 
nus peuvent intervenir sur les phénomènes immédiatement consécutifs à la pénétration: l'activation de l'ovule, vraisemblablement provoquée par une protéine cytosolique du spermatozoïde [15], la formation coordonnée des pronuclei, dont l'asynchronie est préjudiciable au développement [16], la synthèse d'ADN en vue de la première division du zygote, ou l'utilisation optimale des réserves ovulaires pour assurer les trois premiers jours du développement avant le début d'expression du génome embryonnaire [17]. On a observé que la phase S du zygote est plus précoce et plus longue quand le sperme fécondant provient de taureaux sélectionnés pour leur grande fertilité et ce phénomène pourrait être lié, selon les auteurs [18], soit à la qualité de l'activation ovocytaire (pics induits de calcium), soit à la transformation des protéines entourant l'ADN du spermatozoïde (substitution d'histones aux protamines). D'autres observations impliquent une action du pronucleus mâle sur le cytoplasme ovocytaire, action en rapport avec la viabilité du zygote de souris [19]. Il existe donc une relation entre l'issue de la fécondation et la nature du gamète fécondant; dans le cas de l'ICSI, le spermatozoïde fécondant est remarquable par son origine (hommes stériles) mais aussi par le maintien de son intégrité (évitement des modifications induites par la fusion gamétique). De plus, la technique d'injection est susceptible d'améliorer per se le niveau d'activation ovocytaire. Quel qu'en soit le mécanisme, l'affranchissement des barrières ovocytaires augmente l'aptitude au développement. Finalement l'ICSI mérite bien d'être assimilée à un "viol gamétique" [20] tant elle déborde les défenses naturelles de la cellule féminine pour assurer le succès reproductif d'un seul spermatozoïde.

$\mathrm{Au}$ niveau de la grossesse, il est désormais établi qu'un environnement médical adéquat permet de faire naître, sans problème grave, deux enfants simultanément. A condition d'être bien surveillées, les grossesses gémellaires ne sont plus aussi périlleuses qu'elles le furent, et que le demeurent souvent les grossesses que la stimulation du fonctionnement ovarien soit à l'origine de trop nombreuses grossesses multiples (environ $30 \%$ ), l'AMP démontre aussi que la quasi-totalité des femmes sont capables de faire naître deux enfants simultanément, une situation plutôt rare à l'état naturel $(1,6 \%)$ puisque le nombre d'ovules fécondés à chaque cycle est égal ou inférieur à un.

Enfin, par le maintien d'un niveau élevé de prolactine, qui inhibe le fonctionnement ovarien, l'allaitement constitue un procédé élémentaire, mais efficace, de contraception. Ainsi est créée une période réfractaire anovulatoire, capable de différer d'une ou deux années une nouvelle conception. On a reproché au lait artificiel de ne pas transmettre au nouveau-né les anticorps maternels qui lui seraient nécessaires, mais nul n'a évalué la responsabilité de Nestlé dans l'évolution démographique, particulièrement dans le tiers monde !...

\section{En conclusion}

Notre espèce peut aujourd'hui savoir que les handicaps à procréer qu'elle supporte depuis toujours ne sont pas le fait de situations pathologiques mais se révèlent constitutifs des individus "normaux». En effet, les artifices biomédicaux développés pour pallier des déficiences de la fonction procréatrice sont, bien sûr, capables d'annuler les effets inhibiteurs ou de court-circuiter les obstacles responsables de ces déficiences, mais ils peuvent aussi exacerber la fonction au-delà de sa norme.

Il existe donc des inhibitions naturelles de la procréation, agissant à des niveaux variés, principalement: l'âge (empêchement de la procréation juvénile ou sénile), le stock gamétique (gaspillage de 999 ovocytes sur 1000 ), la période fertile (limitée à $10 \%$ de la vie féminine), le comportement (absence de corrélation avec le moment fertile), le rendement ovarien (ovulation unique), la fécondation (pléthore de clés et de serrures)...

En soulignant ces blocages, nous ne prétendons pas proposer de les annuler systématiquement. Le bonheur des individus se suffit de quelques corrections pour recouvrer, si nécessaire, le niveau commun de performance, fût-il dépassable. En ce sens, ce ne sont pas les techniques d'AMP qui devraient inquiéter le plus, malgré leurs dérives (indications abusives, grossesses multiples, devenir des "forces génétiques" congelées, etc.) et leurs innovations incessantes (dernière en date: ICSI). La société s'est alarmée à juste titre de l'utilisation de ces techniques pour augmenter, au-delà du couple, le nombre d'individus impliqués dans la procréation (donneurs de gamètes et embryons, nourrices utérines). Elle doit aussi s'interroger sur les nouvelles possibilités de tri dans l'éprouvette des meilleurs, parmi les nombreux embryons disponibles, grâce au diagnostic génétique préimplantatoire (DPI). Sur ce point, deux publications récentes sont révélatrices. Comme il était prévisible [21], le DPI devient justifiable pour des maladies, certes graves, mais compatibles avec une vie digne d'être vécue (l'hémophilie, par exemple) pourvu que la FIV, indiquée pour infécondité, soit productrice d'embryons surnuméraires [22]; par ailleurs, la culture prolongée de quelques cellules du bouton embryonnaire permet d'obtenir un clone de plusieurs millions de cellules [23], davantage qu'il ne sera jamais nécessaire pour appliquer l'ensemble des sondes génétiques disponibles dans le futur... L'élection d'un enfant de qualité, choisi dès la fécondation, pourrait être considérée comme l'ultime victoire contre les imperfections de la procréation, dont les restrictions multiples évoquées ici ne garantissent cependant pas la «normalité du produit». Ces restrictions, pourvu qu'on leur accorde une signification évolutive, permettent au contraire de multiplier les différences. En effet, si les individus les plus fertiles ne peuvent procréer qu'un nombre limité d'enfants, le poids génétique de leur descendance dans la génération suivante se trouvera relativisé, tandis que d'autres génomes, nombreux et variés, contribueront significativement à cette génération. Les couples totalement empêchés de procréer sont finalement peu nombreux, comme le sont 
ceux dont la progéniture est abondante; le malthusianisme physiologique autorise donc une descendance limitée pour le plus grand nombre d'individus. Ce phénomène restricteur de la compétition génétique joue d'autant plus que la démographie est soumise à des limitations extérieures (ressources alimentaires, libération de la femme, culture, etc.). Ainsi, se trouvent ménagées des ressources génétiques qui auraient pu disparaître dans une compétition exacerbée.

La méiose brasse les gènes pour faire du nouveau à chaque génération, tandis que la procréation autorise la perpétuation du plus grand nombre d'individus, et donc de la plus grande variété de gènes. Les mécanismes évoqués ici font de la procréation une fonction originale puisqu'elle démontre, à chaque phase de son expression, des capacités d'auto-inhibition. Ces mécanismes ne vont pas dans le sens de la théorie du gène " égoïste " et montrent que l'individu n'est pas conformé adéquatement pour assurer son succès reproductif. Il n'est aucune leçon à tirer de ces faits, seulement une conclusion: en matière de procréation, l'inhibition est de règle ; et une hypothèse : cette inhibition cultive la variété

\section{Jacques Testart}

Directeur de recherche à l'Inserm, directeur de l'U. 355 de l'Inserm, 32, rue des Carnets, 92140 Clamart, France.

\section{TIRÉS À PART}

J. Testart.

$m / s n^{\circ} 3$, vol. 11, mars 95

\section{RÉFÉRENCES}

1. Leridon H, Spira A. Problems in measuring the effectiveness of infertility therapy. Fertil Steril $1984 ; 41$ : 580-4

2. Thonneau P, Marchand S, Tallec A, Ferial ML, Ducot B, Lansac J, Lopes $\mathrm{P}$, Tabas te JM, Spira A. Incidence and main causes of infertility in a resident population (1850000) of three French regions (19881989). Hum Reprod $1991 ; 6: 81$ 1-6.

3. Carroll J, Gosden RG. Transplantation of frozen-thawed mouse primordial follicles. Human Reprod 1993; 8:1163-7.

4. Tsunoda $Y$, Tokunaga $T$, Imai $H$, Uchida $T$. Nuclear transplantation of primordial germ cells in the mouse. Development 1989; 107: 407-11.

5. Plachot M, de Grouchy J, Junca AM, Mandelbaum J, Salat-Baroux J, Cohen J. Chromosomal analysis of human oocytes and embryos : does delayed fertilization increase chromosome imbalance? Hum Reprod 1988 $3: 125-7$.

6. Di Zerega GS, Goebelsmann V, Nakamura RM. Identification of protein(s) secreted by the preovulatory ovary which suppresses the follicle response to gonadotropins. $J$ Clin Endocrinol Metab 1982; 54: 1091-6.

7. Boué F, Lassalle B, Duquenne C, Villaroya S, Testart J, Lefevre A, Finaz C. Human sperm proteins from testicular and epididymal origin that participate in fertilization : modulation of sperm binding to zona-free hamster oocytes, using monoclonal antibodies. Mol Reprod Dev 1992; 33: 470-80.

8. Testart J, de Ziegler D. Importance to time gametes meeting in assisted procreation. In: GIFT : from basic to clinics. Serono Symp 63, Raven Press 1989: 245-56.

9. Bleil JD, Wassarman PM. Sperm-egg interactions in the mouse : sequence of events and induction of the acrosome reaction by a zona pellucida glycoprotein. Dev Biol $1983 ; 95$ : 317-24.

10. Lih CH, Grunfeld L, Sandler B, Drews MR, Navot $D$, Gordon JW. Infertile couples with normal counts who require subzonal sperm insertion possess a fertility defect that affects zona pellucida penetration. Hum Reprod 1994; 9: 2335-8.

11. Palermo G, Joris H, Derde MP, Camus M, Devroey $P$, Van Steirteghem A. Sperm characteristics and outcome of human assisted fertilization by subzonal insemination and intracytoplasmic sperm injection. Fertil Steril 1993; 59: 826-35.
12. Ogura A, Matsuda J, Yanagimachi R. Birth of normal young after electrofusion of mouse oocytes with round spermatids. Proc Natl Acad Sci USA 1994; 91 : 7460-2.

13. Van Steirteghem AC, Nagy $Z$, Joris $H$, Liu J, Staessen C, Smitz J, Wisanto A, Devroey $P$. High fertilization and implantation rates after intracytoplasmic sperm injection. Hum Reprod 1993: 8: 1061-6.

14. Bonduelle M, Desmyttere S, Buysse A Van Assch E, Schietecatte J, Devroey P, Van Steirteghem AC, Liebard I. Prospective follows-up study of $\mathbf{5 5}$ children born after subzonal insemination and intracytoplasmic sperm injection. Hum Reprod 1994; 9: 17659.

15. Swann K. A cytosolic sperm factor stimulates repetitive calcium increases and mimics fertilization in hamster eggs. Development 1990; 110: 1295-302.

16. Wright SJ, Longo F J. Sperm nuclear enlargement in fertilized hamster eggs is related to meiotic maturation of the maternal chromatin. J Exp Zool 1988; 247: 155-65.

17. Braude $\mathrm{P}$, Bolton V, Moore S. Human gene expression first occurs between the four- and eight-cell stages of preimplantation development. Nature 1988; 332: 45960.

18. Eid LN, Lorton SP, Parrish JJ. Paternal influence on S-phase in the first cycle of the bovine embryo. Biol Reprod 1994; 51 : 12317.

19. Renard JP, Babinet $\mathrm{CH}$. Identification of a paternal developmental effect on the cytoplasm of one-cell stage mouse embryos. Proc Natl Acad Sci USA 1986; 83: 6883-6.

20. Testart J. L'éprouveur, l'inventreur et le conseilleur. In: La Responsabilité. Paris: Autrement 1994: 276-9.

21. Testart J. Le désir du gène. Paris: François Bourin, 1992.

22. Veiga A, Santalo J, Vidal F, Calderon G, Giménez C, Boada M, Egozcue J, Barri PN. Case report: twin pregnancy after preimplantation diagnosis for sex selection. Hum Reprod 1994; 9: 2156-9.

23. Bongso A, Fong CY, Ng SC, Ratnam S. Isolation and culture of inner cell mass cells from human blastocysts. Hum Reprod 1994 ; $9: 2110-7$. 